\title{
Sensitivity to tonality across the pitch range
}

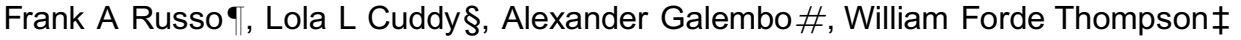 \\ - Department of Psychology, Ryerson University, 350 Victoria Street, Toronto, ON M5B 2K3, Canada; \\ $\S$ Department of Psychology, Queen's University, Kingston, ON K7L 3N6, Canada; \#I M Sechenov \\ Institute of Evolutionary Physiology and Biochemistry, RAS, 44 pr. Morisa Toreza, St Petersburg \\ 194223, Russia; ł Department of Psychology, Macquarie University, Sydney, NSW 2109, Australia; \\ e-mail: russo@ryerson.ca \\ Received 2 February 2005, in revised form 18 June 2006; published online 27 April 2007
}

\begin{abstract}
Striking changes in sensitivity to tonality across the pitch range are reported. Participants were presented a key-defining context (do-mi-do-sol) followed by one of the 12 chromatic tones of the octave, and rated the goodness of fit of the probe tone to the context. The set of ratings, called the probe-tone profile, was compared to an established standardised profile for the Western tonal hierarchy. The presentation of context and probe tones at low and high pitch registers resulted in significantly reduced sensitivity to tonality. Sensitivity was especially poor for presentations in the lowest octaves where inharmonicity levels were substantially above the threshold for detection. We propose that sensitivity to tonality may be influenced by pitch salience (or a co-varying factor such as exposure to pitch distributional information) as well as suprathreshold inharmonicity.
\end{abstract}

\section{Introduction}

Sensitivity to musical tonality includes sensitivity to the hierarchical organisation of tones about a reference tone. This organisation has been formalised, both by music theory (eg Lerdahl 2001; Piston 1987) and by empirical study (eg Krumhansl 1990, 2000; Toiviainen and Krumhansl 2003), as the tonal hierarchy - a hierarchy of stability and salience of tones. Tonal hierarchies occur in the music of many cultures (Castellano et al 1984; Krumhansl et al 2000; Lantz et al 2003); the present paper focuses on the tonal hierarchy of Western-harmonic music. Western-harmonic music has been characterised by scale systems, in particular the diatonic system, do-re-mi-fa-sol-la-ti-do. In the diatonic system, the highest stability is associated with the tonic tone (do), followed by non-tonic triad tones (mi, sol), followed by non-triadic tones (re, fa, la, ti), and finally the non-scale tones.

Krumhansl and Kessler (1982) demonstrated empirically that listeners are sensitive to this hierarchy of stability. They obtained ratings of the goodness of fit of probe tones following a number of key-defining contexts. All tones in the chromatic scale were used as probe tones. Standardised profiles for the major and minor keys were derived by averaging probe-tone ratings across listeners and across several key-defining contexts. These profiles revealed a hierarchy of probe-tone ratings consistent with music-theoretic descriptions of tonal stability.

Sensitivity to tonality is dependent on a number of perceptual and cognitive factors that give rise to individual differences in profiles across listeners. For example, probetone ratings can be influenced by the listener's degree of music training (Krumhans1 and Shepard 1979) and age (Cuddy and Badertscher 1987; Krumhansl and Keil 1982; Speer and Meeks 1985). Sensitivity to tonality can be impaired following brain damage (Steinke et al 2001) and in some cases may be dissociated from other musical abilities (Peretz 2001; Peretz et al 1994). Psychological investigations of tonality and harmony have also considered and in some cases modeled potential influences on psychoacoustic factors (eg Leman 2000; Thompson and Parncutt 1997). 
Such psychoacoustic models imply that influences on sensitivity to tonality operate at relatively early stages of pitch processing. With musical tones, pitch proximity between the probe tone and the final tone of a key-defining context influences probetone ratings (Krumhansl and Shepard 1979). Probe tones near in pitch distance to the context are rated higher than probe tones further in pitch distance. To avoid potential influences of pitch distance on the standardised tonal hierarchy, Krumhansl and Kessler (1982) used circular tone timbres. All tones were constructed by combining nine octave-spaced frequencies, with the amplitude of each component determined by a fixed amplitude envelope. A consequence of using circular tones is that the influence of pitch distance is suppressed.

Although the effect of pitch distance between context and probe tone is acknowledged, the overall effect of pitch height on sensitivity to tonality is unknown. The present investigation addresses the issue. Sensitivity to tonality was assessed in 15 overlapping octaves that spanned the range from the $\mathrm{E}^{b}$ below the lowest $\mathrm{C}\left(\mathrm{E}_{0}^{b}, 19.5 \mathrm{~Hz}\right)$ to the highest $\mathrm{C}\left(\mathrm{C}_{8}, 4186 \mathrm{~Hz}\right)$ of the standard piano pitch range. The lowest five octaves are referred to as the low-pitch region (bass), the middle five octaves as the middle-pitch region (tenor), and the highest five octaves as the high-pitch region (treble).

Sensitivity was assessed with the probe-tone method (Krumhansl 1990; Krumhansl and Kessler 1982; Krumhansl and Shepard 1979). The standardised tonal hierarchy documented by Krumhansl and Kessler (1982) was used as a referent against which sensitivity to tonality was estimated. Accordingly, the correlation between a profile obtained under a given experimental condition and the standardised profile was treated as a measure of sensitivity to tonality. A strong correlation reflects better recovery of the tonal hierarchy and hence greater sensitivity to tonality.

A number of factors led us to predict that sensitivity to tonality would vary across the pitch range: the degree to which inharmonicity levels in tones exceed the threshold for detection; pitch salience; and long-term exposure to the distribution of pitches in music. To implement the predictions, we used one popular keyboard instrument, the Roland FP-1. Piano sounds (sampled in the case of the FP-1) exemplify the properties of struck or plucked strings; they possess inharmonicity across the tessitura. (Not all instruments possess inharmonicity.) In addition, the range of the piano, which encompasses most orchestral instruments, is sufficiently broad to allow predictions based on pitch salience and long-term exposure to distributions to be tested. The Roland FP-1 was specifically chosen because it is a MIDI instrument and as such may be easily controlled, and because it produces inharmonic tones based on digitised samples taken across the tessitura of a real piano.

\subsection{Inharmonicity}

The presence of inharmonicity in tones influences the perception of tonal relations and it may reduce sensitivity to tonality. Cohen (1984) found that participants had greater difficulty tuning common musical intervals (fifth, octave) when the synthetic tones used for tuning were inharmonic rather than harmonic. Mathews and Pierce (1980) compared key matching for melodic passages composed of inharmonic synthetic tones with key matching for melodic passages composed of harmonic synthetic tones. They found greater matching difficulty for passages containing inharmonic synthetic tones. Moreover, they found that, for chord pairs forming either a perfect cadence (dominant to tonic) or an imperfect cadence (tonic to dominant), chord pairs composed of inharmonic synthetic tones were judged to convey an equal degree of finality regardless of cadence type. In contrast, judgments of chord pairs composed of harmonic synthetic tones assigned greater finality to the perfect as opposed to the imperfect cadence - a finding consistent with traditional music theory. 
The reduced sensitivity to tonality observed with synthetic inharmonic tones may result from a degradation of pitch perception. Degraded pitch perception may compromise the extraction of pitch relations, which, in turn, may lead to decreased sensitivity to tonality. Theoretical models of pitch can account for degraded pitch perception observed when inharmonicity levels exceed the threshold for detection. First, spectral models of pitch perception predict optimal pitch perception when partials fall along the harmonic series and non-optimal pitch perception when tones contain stretched partials (Terhardt 1974; Wightman 1973). Second, temporal mechanisms involved in the formation of residue pitch favour periodic complex tones, whereas aperiodicity arising from inharmonicity may disrupt residue pitch (Schouten et al 1962). Third, inharmonicity gives rise to phase randomisation, thus diminishing peak structure in the waveform (Galembo et al 2001) and interfering with temporal mechanisms involved in pitch perception (Patterson 1973; Ritsma 1962; Schouten et al 1962).

The presence of inharmonicity tends to vary as a function of fundamental frequency. As frequency increases, inharmonicity levels in most pianos decrease initially but then increase rapidly throughout the upper frequency region (Conklin 1999; Fletcher 1964; Fletcher et al 1962; Galembo 1987). The threshold for detecting inharmonicity also varies as a function of frequency, such that the presence of inharmonicity in the lower pitch region is most likely to be detected. Järveläinen et al (2001) determined inharmonicity thresholds as a function of fundamental frequency for synthesised tones with spectral properties in common with piano tones. They found that inharmonicity was easiest to detect in the low-pitch region. That is, although absolute levels of inharmonicity were highest in the high-pitch region, levels of inharmonicity significantly above the detection threshold were highest in the low-pitch region (also see Fletcher et al 1962; Rocchesso and Scalcon 1999). In view of these results, we expected that inharmonicity might contribute to reduced sensitivity to tonality in the low-pitch region.

\subsection{Pitch salience}

Terhardt et al (1982) proposed a model of pitch perception that allows for the computation of pitch salience. According to this model, the salience of the pitch of a complex tone depends on spectral dominance, masking, and subharmonic coincidence. ${ }^{(1)}$ In complex tones, the relation between fundamental frequency and pitch salience follows an inverted- $\mathrm{U}$ function centred at about $300 \mathrm{~Hz}$ (approximately 2 semitones above middle $C$ ). When very low or very high pitches are heard, the model generates multiple pitch candidates, which weakens pitch salience. The pitch salience of pure tones (defined as pitch strength) has also been measured and yields a similar inverted-U function (Fastl 1989). However, the function for pure tones is centred about 2 octaves above the computed function for complex tones. This difference is attributable to the lack of overtone structure in pure-tone stimuli.

\subsection{Exposure to pitch distributional information}

Meyer (1956) argued that, through long-term exposure to music, listeners internalise statistical regularities and interpret new musical input with reference to this tacit knowledge. Krumhansl (1990) later proposed that sensitivity to tonality reflects knowledge of pitch distributional information, which is internalised automatically and unconsciously through exposure to music. She demonstrated that the frequency of occurrence of pitches (collapsed across octaves) in a large corpus of Western music maps closely onto the standard tonal hierarchy. Oram and Cuddy (1995) found that ratings of the goodness of fit of probe tones following melodic contexts may also be influenced by pitch distributional information contained in the context itself.

(1) The determination of subharmonic coincidence is influenced in part by the inharmonicity of a tone (ie more inharmonicity leads to fewer coincidences) but does not take into account the threshold of inharmonicity. 
Tones in the middle region occur more frequently and are likely to be heard more often than tones at the extremes of the tessitura (low-pitch and high-pitch regions). This increased frequency of occurrence in the middle-pitch region should lead to increased familiarity with such tones and processing advantages for them. Evidence for the influence of familiarity on pitch processing has been demonstrated for absolutepitch identification and expectancy judgments. White notes occur more often than black notes on the piano and are identified more accurately than black notes by absolute pitch possessors (Takeuchi and Hulse 1991). Data on melodic expectancy suggest that listeners expect pitches to fall in the middle of the tessitura (von Hippel 2000; von Hippel and Huron 2000).

Figure 1 shows plots of standardised values of pitch salience for piano tones used in this study and the frequency of occurrence of pitches in real keyboard music. The pitch salience values were calculated according to the Terhardt-Stoll-Seewann model (Terhardt et al 1982). This model predicts that pitch salience of a complex tone will vary as a function of both fundamental frequency and inharmonicity. The frequencyof-occurrence values were derived by performing counts for notes within each octave in a sample of keyboard music. The sample consisted of 100 MIDI transcriptions (Schwob 1999) of popular pieces for keyboard instruments composed in the Westerncommon-practice era (eg Mozart, Beethoven, Schumann). Perhaps not surprisingly, the correlation between these two functions was almost perfect $\left(r_{13}=0.97, p<0.0001\right)$. We expected that sensitivity to tonality would be strongest in the region where pitch salience was highest and where pitches occur most frequently.

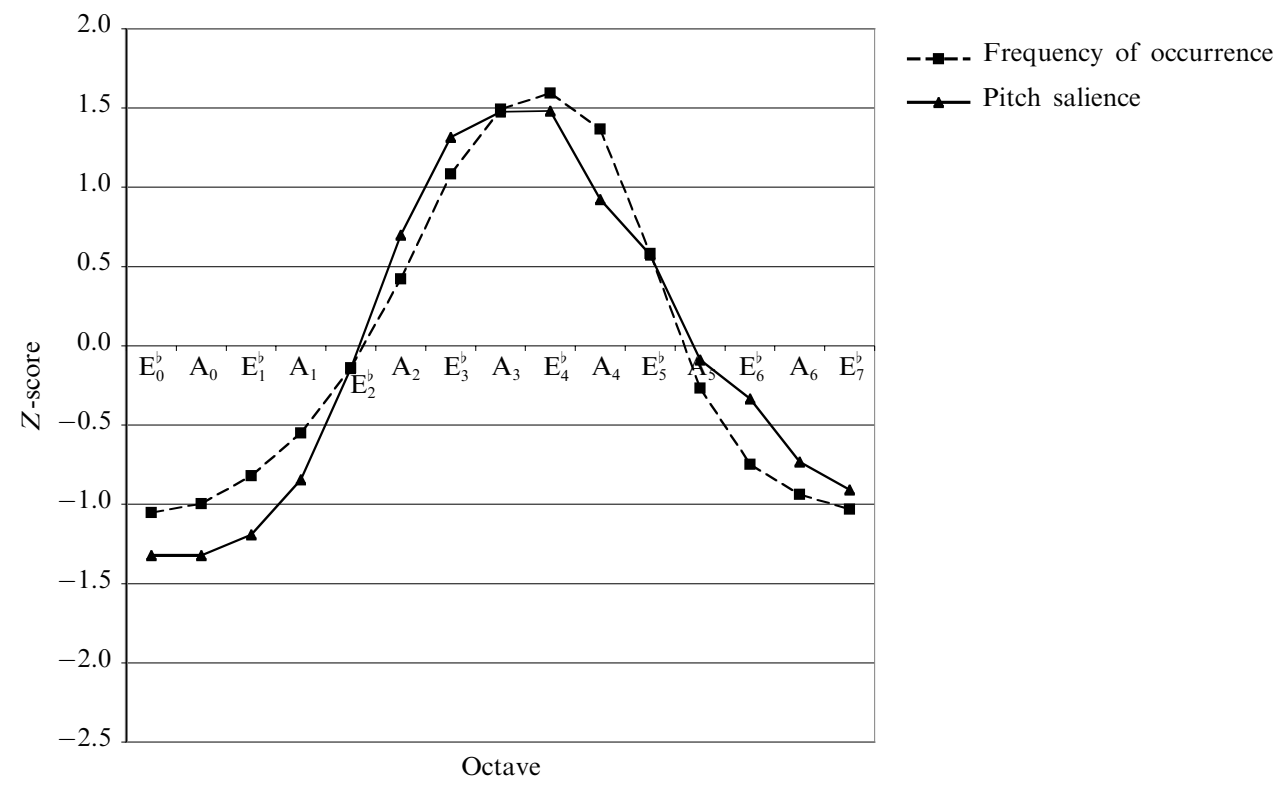

Figure 1. $Z$-score plot of the frequency of occurrence of pitches in Western keyboard music and the computed pitch salience values for piano tones stimuli.

\section{Method}

\subsection{Participants}

Twenty participants (fourteen females and six males) were recruited from the Queen's University community. All participants were highly trained in music. Music training was assessed by a point system. One point was awarded for each year of private instruction and a half point was awarded for each year of group instruction. (Points were not double in cases of instruction on two instruments within the same year.) 
Participants had a minimum of 9 points, a mean of 12.45 points $(\mathrm{SE}=0.65$ ), and typically had experience with two instruments and some continued activity in music beyond casual listening. Participants varied in age from 18 to 26 years with a mean age of 21.6 years. All participants were given course credit for their participation. No participant reported having abnormal hearing or absolute pitch.

\subsection{Materials}

Stimuli were generated by a Roland FP-1 digital piano under the control of a Macintosh Power PC. Tones were presented in a sound-attenuated chamber over Roland FPS-1 stand speakers. Test sequences were presented in each of 15 octaves. The pitch range of each octave and the corresponding range of fundamental frequencies are described in table 1. A musical context, the sequence of tones do-mi-do-sol (after Cuddy and Badertscher 1987; Krumhansl and Keil 1982), was presented, followed by one of the 12 chromatic tones of the octave. Duration of each tone was $0.33 \mathrm{~s}$. The last tone of the context and the probe tone were separated by an interval of $1 \mathrm{~s}$. Loudness of all tones was equalised on the basis of average loudness matching by five expert listeners. Intensity of the standard tone $\left(\mathrm{A}_{4}\right)$ for loudness matching was $70 \mathrm{~dB}$ SPL.

Table 1. Pitch and frequency range for each octave tested.

\begin{tabular}{llllllllll}
\hline Octave & Pitch & Range & $F_{0}$ & Range $/ \mathrm{Hz}$ & Octave & Pitch & Range & $F_{0}$ & Range $/ \mathrm{Hz}$ \\
\hline 1 & $\mathrm{E}_{0}^{b}$ & $\mathrm{D}_{1}$ & 19.5 & 36.7 & 9 & $\mathrm{E}_{4}^{b}$ & $\mathrm{D}_{5}$ & 311.1 & 587.3 \\
2 & $\mathrm{~A}_{0}$ & $\mathrm{G}_{1}^{\sharp}$ & 27.5 & 51.9 & 10 & $\mathrm{~A}_{4}$ & $\mathrm{G}_{5}^{\sharp}$ & 440.0 & 830.6 \\
3 & $\mathrm{E}_{1}^{b}$ & $\mathrm{D}_{2}$ & 38.9 & 73.4 & 11 & $\mathrm{E}_{5}^{b}$ & $\mathrm{D}_{6}$ & 622.3 & 1174.7 \\
4 & $\mathrm{~A}_{1}$ & $\mathrm{G}_{2}^{\sharp}$ & 55.0 & 103.8 & 12 & $\mathrm{~A}_{5}$ & $\mathrm{G}_{6}^{\sharp}$ & 880.0 & 1661.2 \\
5 & $\mathrm{E}_{2}^{b}$ & $\mathrm{D}_{3}$ & 77.8 & 146.8 & 13 & $\mathrm{E}_{6}^{b}$ & $\mathrm{D}_{7}$ & 1244.5 & 2349.3 \\
6 & $\mathrm{~A}_{2}$ & $\mathrm{G}_{3}^{\sharp}$ & 110.0 & 207.7 & 14 & $\mathrm{~A}_{6}$ & $\mathrm{G}_{7}^{\sharp}$ & 1760.0 & 3322.4 \\
7 & $\mathrm{E}_{3}^{b}$ & $\mathrm{D}_{4}$ & 155.6 & 293.7 & $15^{(a)}$ & $\mathrm{D}_{7}^{b}$ & $\mathrm{C}_{8}$ & 2217.5 & 4186.0 \\
8 & $\mathrm{~A}_{3}$ & $\mathrm{G}_{4}^{\sharp}$ & 220.0 & 415.3 & & & & &
\end{tabular}

(a) Because $\mathrm{C}_{8}$ is the upper limit of pitch on the Roland FP-1, the 11th and 12th probes in the 15th octave were $\mathrm{D}_{7}^{b}$ and $\mathrm{D}_{7}$ respectively (ie below the tonic $\left[\mathrm{E}_{7}^{b}\right]$ of the implied key).

\subsection{Probe-tone procedure}

Participants received 180 trials, representing 12 probe tones in each of the 15 octaves tested. The task was to rate the degree to which the probe tone fit the context on a 7-point scale that ranged from "fits very poorly" to "fits very well". Participants were encouraged to assess the probe tone with respect to the entire 4-tone context rather than on how well the probe tone continued the melody. The order of trials was independently randomised for each participant.

\subsection{Acoustical measurements}

Every third tone from $\mathrm{E}_{0}^{b}$ to $\mathrm{A}_{7}$ produced by the Roland FP-1 was subjected to acoustical analysis. The first $0.3 \mathrm{~s}$ of each tone was recorded and sampled at $44 \mathrm{kHz}$ (16 bit) with a Digi 002 analog-to-digital converter. Digitised samples were stored as wave files (.wav). A fast-Fourier transformation was applied to each digitised waveform and the resulting spectra were analysed with Praat software (Boersma and Weenink 2004). ${ }^{(2)}$ For each tone, the frequency of all prominent partials beyond the fifth partial was entered into a vector. A second vector of frequencies with the same number of entries

(2) The reported measurements were obtained by the first author. To verify the accuracy of these measurements, a subset of eight original recordings $\left(\mathrm{A}_{0}, \mathrm{~A}_{1}, \mathrm{~A}_{2}, \mathrm{~A}_{3}, \mathrm{~A}_{4}, \mathrm{~A}_{5}, \mathrm{~A}_{6}\right.$, and $\left.\mathrm{A}_{7}\right)$ was analysed by the third author. The method of analysis was identical; however, the software used for spectral analysis was Cool Edit rather than Praat. There was an extremely high level of agreement between the two sets of corresponding measurements $\left(r_{6}=0.99, p<0.0001\right)$. 
was generated with the standard formula for string inharmonicity (Fletcher et al 1962). Values of $B$ (the coefficient of inharmonicity; higher values reflect more inharmonicity) and $f_{0}$ (fundamental frequency) that minimised the least-squares difference between the two vectors were determined.

Figure 2 is a logarithmic plot of the measured inharmonicity values for tones produced by the FP-1 and the threshold inharmonicity values determined by Järveläinen et al (2001). Although measured values were consistently above threshold values, this difference decreased as a function of increasing frequency. For the pitch region above $\mathrm{A}_{2}$, the observed trend in harmonicity for tones produced by the Roland FP-1 was highly similar to observed trends in tones produced by real acoustic pianos (eg Fletcher 1964; Fletcher et al 1962). However, the inharmonicity trend for tones below $\mathrm{A}_{2}$ was atypical. As seen in figure 2 , the function is flat below $\mathrm{A}_{2}$, with $B$ values ranging from 0.00008 to 0.0001 . In contrast, real pianos tend to exhibit increasing inharmonicity with decreasing frequency below $\mathrm{A}_{2}$. Examination of the waveform of FP-1 tones in this range revealed that the same sample was used for all tones. This finding suggests that a piano tone possessing low inharmonicity $\left(\right.$ eg $\left.\mathrm{G}_{2}\right)$ was digitally recorded and then upsampled to produce appropriate equal-tempered pitches down to $\mathrm{E}_{0}^{b}$. This method generated lower inharmonicity levels than would be realised had an independent sample been taken for each tone.

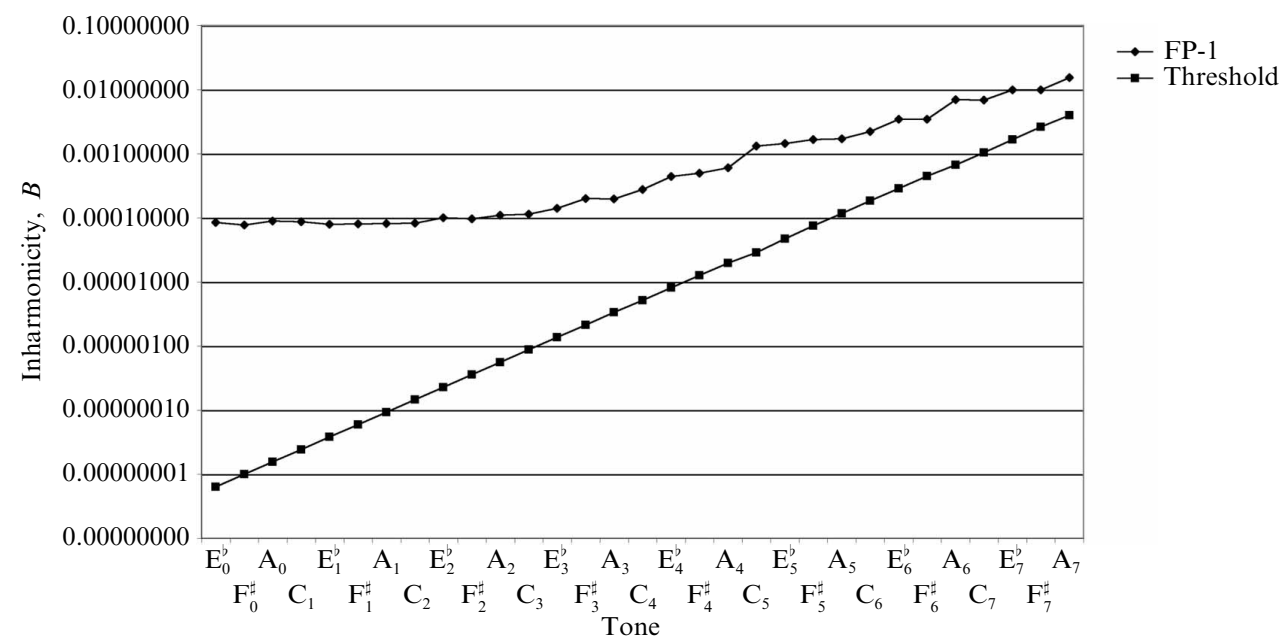

Figure 2. Logarithmic plot of the measured inharmonicity values for tones produced by the FP-1 and the threshold inharmonicity values determined by Järveläinen et al (2001).

\section{Results and discussion}

Figure 3 displays the standardised major-key profile along with mean ratings for each of the 12 probe tones in two representative octaves. The profiles are taken from octaves in the middle-pitch and low-pitch regions $\left(\mathrm{A}_{3}\right.$ and $\mathrm{A}_{0}$, respectively) in order to illustrate the effect of pitch region on sensitivity to tonality. The correlation between the standardised major-key profile and the octave in the middle-pitch region was higher than the correlation between the standardised profile and the octave in the low-pitch region $\left(r_{10}=0.95\right.$ and 0.79$)$. Mean ratings for the octave in the middle-pitch region clearly illustrate the four-level hierarchy characteristic of the standardised profile. Mean ratings for the octave in the low-pitch region show less differentiation and do not clearly illustrate the four-level hierarchy. Nonetheless, it is instructive to note that both correlations were significant $(p<0.05)$, and thus some level of sensitivity to tonality does still exist in the low-pitch region. 


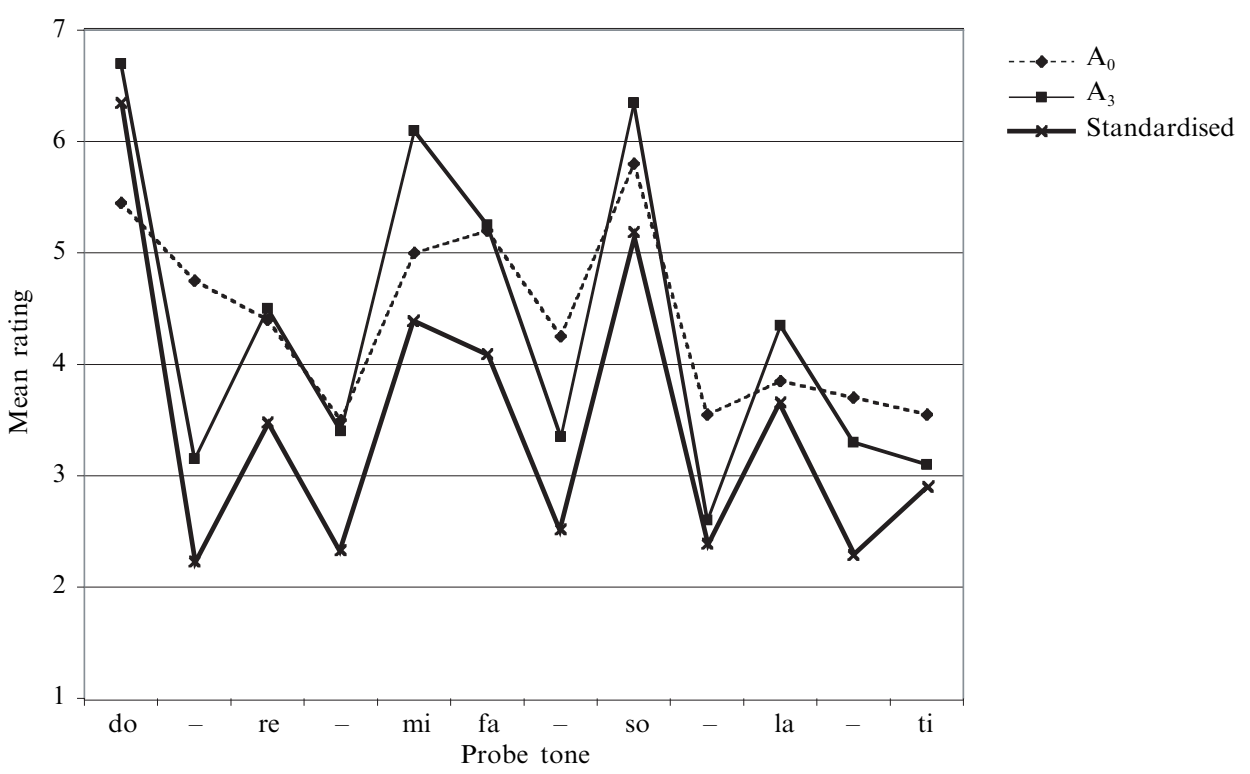

Figure 3. The standardised major-key profile along with mean ratings for each of the 12 probe tones in the $\mathrm{A}_{0}$ and $\mathrm{A}_{3}$ octaves.

For each participant, the observed probe-tone profile for each of the 15 octaves was correlated with the standardised major-key profile resulting in 15 recovery scores (ie correlation coefficients). The distribution of recovery scores within each octave was approximately normal. Recovery scores were subjected to an analysis of variance with range as the within-subjects factor. Recovery scores increased progressively in octaves $E_{0}^{b}$ through $A_{1}$ but then decreased again slightly beyond $A_{5}$. Figure 4 shows a plot of the best-fitting polynomial regression line for recovery scores.

Enhanced tonality perception in the middle-pitch region may reflect pitch salience. However, reduced tonality perception was more evident in the low-pitch region than in the high-pitch region. This asymmetry cannot be explained entirely by pitch salience

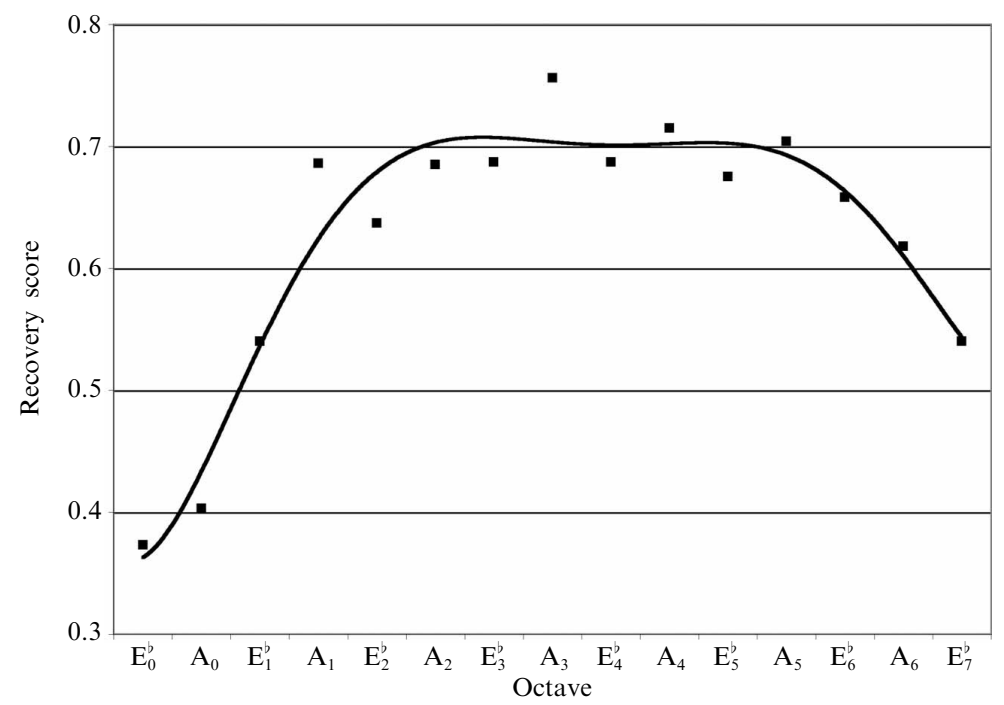

Figure 4. Plot of the best-fitting polynomial regression line (6th order) for mean recovery scores (squares). 
(or co-varying factors such as exposure to the distribution of pitches in music), as that distribution is symmetric and centred on the middle of the tessitura. To explore this asymmetry, we compared acoustic measurements of the degree of inharmonicity in the tones used with threshold values for inharmonicity. The suprathreshold inharmonicity level was determined for each octave by subtracting threshold inharmonicity from measured inharmonicity (see figure 1). The combined predictions from suprathreshold inharmonicity and pitch salience account for the asymmetric recovery function displayed in figure 4. A multiple-regression analysis revealed that both predictors contributed significantly, accounting for $71 \%$ of variability in mean recovery scores $\left(F_{2,14}=14.99, p<0.001\right)$. A model incorporating suprathreshold inharmonicity and pitch distributional information accounted for the identical proportion of variance $\left(F_{2,14}=14.58, p<0.001\right)$ - see table 2 for details.

Table 2. Standardised beta weights $(B)$ for regression models that predict mean recovery scores from pitch salience and suprathreshold inharmonicity (left), and pitch distributional information and suprathreshold inharmonicity (right).

\begin{tabular}{|c|c|c|c|c|c|c|c|}
\hline Predictor & $B$ & $T$ & $p$ & Predictor & $B$ & $T$ & $p$ \\
\hline Pitch salience & 0.63 & 3.72 & 0.01 & $\begin{array}{l}\text { Pitch distributional } \\
\text { information }\end{array}$ & 0.59 & 3.65 & 0.01 \\
\hline $\begin{array}{l}\text { Suprathreshold } \\
\text { inharmonicity }\end{array}$ & -0.37 & -2.19 & 0.05 & $\begin{array}{l}\text { Suprathreshold } \\
\text { inharmonicity }\end{array}$ & -0.48 & -2.97 & 0.05 \\
\hline $\begin{array}{l}\text { Overall model: } \\
\qquad R=0.85 ; F_{2,14}\end{array}$ & 4.99, $p$ & 0.001 . & & $\begin{array}{l}\text { Overall model: } \\
\qquad R=0.84 ; F_{2,14}=\end{array}$ & $.58,1$ & 0.001 & \\
\hline
\end{tabular}

The poor recovery scores in the low-pitch region may be attributed to high levels of suprathreshold inharmonicity in this frequency range, along with low pitch salience. ${ }^{(3)}$ Beyond this range, recovery remained relatively stable until the drop in sensitivity beyond the $\mathrm{A}_{5}$ octave. Reduced sensitivity in the high-pitch region may be due to low levels of pitch salience. The reduced sensitivity in both the low-pitch and high-pitch regions may also be due to low levels of familiarity. Again, it is not possible to disambiguate the relative influence of familiarity and pitch salience because these trends co-vary.

It is likely that similar asymmetric recovery functions would be observed for other instruments, but recovery functions are unlikely to be identical for different instruments. First, inharmonicity levels vary for different instruments (Fletcher and Rossing 1998). For example, inharmonicity is nonexistent in tones produced by non-stringed instruments (eg flute) and for stringed instruments that are bowed rather than struck (Brown 1996). Second, different instruments are associated with different pitch distributions. Any timbre-related influence of familiarity on sensitivity to tonality, however, is probably quite limited. The process of internalising musical regularities is presumed to depend on abstractions of pitch information from the musical surface (Krumhansl 1990;

(3) To further verify the influence of inharmonicity on tonal sensitivity in the low-pitch region of the piano, we conducted a simple experiment in which inharmonicity was independently varied. All tones were in the $\mathrm{A}_{0}$ octave, and had piano-like spectral shape and bandwidth. Tones in an inharmonic condition had inharmonicity levels that adhered to a power function $\left(B=0.013 f_{0}^{-1}\right)$ described by Rasch and Heetvelt (1985), with levels falling above threshold but not exceeding observed levels for acoustic pianos (Conklin 1999; Fletcher 1964; Fletcher et al 1962). Tones in a harmonic condition had no inharmonicity $(B=0)$. The correlation of the standard major profile with the average profile was higher in the harmonic than in the inharmonic condition $\left(r_{10}=0.94\right.$ and 0.69$)$. In addition, recovery scores were significantly higher in the harmonic condition (mean $=0.61, \mathrm{SE}=0.06)$ than in the inharmonic condition (mean $=0.17, \mathrm{SE}=0.11$ ) $\left(F_{1,11}=20.30, p<0.01\right)$. 
Meyer 1956). There is no obvious reason for an abstraction of pitch information to retain timbral information. Moreover, most listeners do not have difficulty recovering the tonal hierarchy from contexts composed of tones with highly unfamiliar timbres such as pure or circular tones (eg Krumhansl 1979; Krumhansl and Kessler 1982).

\section{Conclusions}

The current data reveal that sensitivity to tonality changes significantly across the tessitura, with dramatically reduced sensitivity to tonality in lower pitch regions and moderately reduced tonality perception in upper pitch regions. Such an asymmetric sensitivity function may result from the combined influence of pitch salience (or a co-varying factor such as exposure to pitch distributional information in music) and reduced pitch processing that occurs when inharmonicity levels exceed the threshold of detection.

The variability in sensitivity to tonality observed in this study has important implications for composition, orchestration, and music analysis. Melodies that are scored in the low-pitch region of the piano may give rise to weak tonal implications, for example. Musical conventions that strongly establish a tonal centre in the middlepitch region (eg a perfect cadence) may only weakly establish a tonal centre in the low-pitch region. When music is composed at the upper and lower boundaries of the tessitura, tonal implications may be fragile, allowing other qualities such as rhythm and timbre to dominate our experience of the music.

Acknowledgments. This research was supported by a Discovery grant from the Natural Sciences and Engineering Research Council of Canada awarded to the second author and a NATO Postdoctoral Science Fellowship awarded to the third author. We are grateful to Jung-Kyong Kim for running participants and to Jason Cullimore for assistance with calculating frequency of occurrence of pitches in real keyboard music.

\section{References}

Boersma P, Weenink D, 2004 "Praat: Doing phonetics by computer" available at http://www.praat.org/ Brown J C, 1996 "Frequency ratios of spectral components of musical sounds" Journal of the Acoustical Society of America $991210-1218$

Castellano M A, Bharucha J J, Krumhansl C L, 1984 "Tonal hierarchies in the music of North India" Journal of Experimental Psychology: General $113394-412$

Cohen E A, 1984 "Some effects of inharmonic partials on interval perception" Music Perception $1323-349$

Conklin H A, 1999 "Generation of partials due to nonlinear mixing in stringed instruments" Journal of the Acoustical Society of America 105 536-545

Cuddy L L, Badertscher B, 1987 "Recovery of the tonal hierarchy: Some comparisons across age and levels of musical experience" Perception \& Psychophysics $41609-620$

Fastl H, 1989 "Pitch strength of pure tones" Proceedings of the 13th International Congress on Acoustics, Belgrade Ed. P Pravica (Šabac, Yugoslavia: Dragan Srnic)() pp 11 - 14

Fletcher H, 1964 "Normal vibration frequencies of a stiff piano string" Journal of the Acoustical Society of America $36203-209$

Fletcher H, Blackham E D, Stratton R, 1962 "Quality of piano tones" Journal of the Acoustical Society of America $34749-761$

Fletcher N H, Rossing T D, 1998 The Physics of Musical Instruments (New York: Springer)

Galembo A, 1987 The Quality of Piano Tones (Moscow: Legkaya Industriya) [in Russian]

Galembo A, Askenfelt A, Cuddy L L, Russo F A, 2001 "Effects of relative phases on pitch and timbre in the piano bass range" Journal of the Acoustical Society of America $1101649-1666$

Hippel P von, 2000 "Redefining pitch proximity: Tessitura and mobility as constraints on melodic intervals" Music Perception 17315 - 327

Hippel P von, Huron D, 2000 "Why do skips precede reversals? The effect of tessitura on melodic structure" Music Perception 18 59-85

Järveläinen H, Valimaki V, Karjalainen M, 2001 "Audibility of the timbral effects of inharmonicity in stringed instrument tones" Acoustics Research Letters Online 2 79-84

Krumhansl C L, 1979 "The psychological representation of musical pitch in a tonal context" Cognitive Psychology 11 346-374

Krumhansl C L, 1990 Cognitive Foundations of Musical Pitch (New York: Oxford University Press) 
Krumhansl C L, 2000 "Rhythm and pitch in music cognition" Psychological Bulletin 126159 - 179

Krumhansl C L, Keil F C, 1982 "Acquisition of the hierarchy of tonal functions in music" Memory \& Cognition $10243-251$

Krumhansl C L, Kessler E J, 1982 "Tracing the dynamic changes in perceived tonal organization in a spatial representation of musical keys" Psychological Review 89 334-368

Krumhansl C L, Shepard R N, 1979 "Quantification of the hierarchy of tonal functions within a diatonic context" Journal of Experimental Psychology: Human Perception and Performance $5579-594$

Krumhansl C L, Toivanen P, Eerola T, Toivianinen P, Järvinen T, Louhivuori J, 2000 "Cross-cultural music cognition: Cognitive methodology applied to North Sami yoiks" Cognition $7613-58$

Lantz M E, Kim J, Cuddy L L, 2003 "The influence of tone duration on pitch structure: Perception of a Korean tonal hierarchy" paper presented at the Society for Music Perception and Cognition, University of Las Vegas, Nevada

Leman M, 2000 "An auditory model of the role of short-term memory in probe-tone ratings" Music Perception 17481 - 509

Lerdahl F, 2001 Tonal Pitch Space (New York: Oxford University Press)

Mathews M V, Pierce J R, 1980 "Harmony and nonharmonic partials" Journal of the Acoustical Society of America $\mathbf{6 8} 1252-1257$

Meyer L B, 1956 Emotion and Meaning in Music (Chicago: University of Chicago Press)

Oram N, Cuddy L L, 1995 "Responsiveness of Western adults to pitch distributional information in melodic sequences" Psychological Research/Psychologische Forschung 57 103-118

Patterson R D, 1973 "The effects of relative phase and the number of components on residue pitch" Journal of the Acoustical Society of America 53 1565-1572

Peretz I, 2001 "Music perception and recognition", in The Handbook of Cognitive Neuropsychology: What Deficits Reveal About the Human Mind Ed. B Rapp (Philadelphia, PA: Taylor \& Francis) pp $519-540$

Peretz I, Kolinsky R, Tramo M, Labrecque R, Hublet C, Demeurisse G, Belleville S, 1994 "Functional dissociations following bilateral lesions of auditory cortex" Brain 117 1238-1301

Piston W, 1987 Harmony 5th edition (New York: W W Norton \& Co.)

Rakowski A, Miskiewicz A, 2002 "Pitch discrimination of low-frequency tones" Proceedings of the 7th International Conference on Music Perception and Cognition, Sydney Eds C Stevens, D Burnham, G McPherson, E Schubert, J Renwick (Adelaide, Australia: Causal Production) pp $538-540$

Rasch R A, Heetvelt V, 1985 "String inharmonicity and piano tuning" Music Perception 3171 - 190

Ritsma R J, 1962 "Existence region of the tonal residue: Part I" Journal of the Acoustical Society of America $341224-1229$

Rocchesso D, Scalcon F, 1999 "Bandwidth of perceived inharmonicity for physical modeling of dispersive strings" IEEE Transactions on Speech and Audio Processing 7 597-601

Schouten J F, Ritsma R J, Cardozo B L, 1962 "Pitch of the residue" Journal of the Acoustical Society of America 341418 - 1424

Schwob P R, 1999 "Classical MIDI archives", available at http:///www.prs.net/midi.html

Speer J R, Meeks P U, 1985 "School children's perception of pitch in music" Psychomusicology 5 $49-56$

Steinke W R, Cuddy L L, Jakobson L S, 2001 "Dissociations among functional subsystems governing melody recognition after right-hemisphere damage" Cognitive Neuropsychology 18 $411-437$

Takeuchi A H, Hulse S H, 1991 "Absolute pitch judgments of black- and white-key pitches" Music Perception $927-46$

Terhardt E, 1974 "Pitch, consonance, and harmony" Journal of the Acoustical Society of America 551061 - 1069

Terhardt E, Stoll G, Seewann M, 1982 "Pitch of complex signals according to virtual-pitch theory: Test, examples, and predictions" Journal of the Acoustical Society of America 71 671-678

Thompson W F, Parncutt R, 1997 "Perceptual judgements of triads and dyads: Assessment of a psychoacoustic model" Music Perception $14263-280$

Toiviainen P, Krumhansl C L, 2003 "Measuring and modeling real-time responses to music: The dynamics of tonality induction" Perception $32741-766$

Wightman F L, 1973 "The pattern-transformation model of pitch" Journal of the Acoustical Society of America $\mathbf{5 4} 407-416$ 


\section{PERTEPTION}

VOLUME 362007

www.perceptionweb.com

Conditions of use. This article may be downloaded from the Perception website for personal research by members of subscribing organisations. Authors are entitled to distribute their own article (in printed form or by e-mail) to up to 50 people. This PDF may not be placed on any website (or other online distribution system) without permission of the publisher. 